\title{
Interatomic Coulombic decay: a short review
}

\author{
AxM Dias* \\ Universidade José do Rosário Vellano, UNIFENAS \\ Caixa Postal 23, CEP 37130-00 Alfenas, MG. Brasil
}

(Received on 14 November, 2008)

\begin{abstract}
The transition process of the interatomic Coulombic decay (ICD), is an electronic radiationless transition process, driving molecular complexes or clusters to a doubly ionized final state. This process differs from the Auger effect, because it takes place from a neutral monomer after the absorption of a released amount energy of the neighboring monomer in the weakly bound molecule. This process has been theoretically studied and the most recent experimental evidence was observed with neon dimer. This work presents a description of the process and a detailed revision of the derivation for the distribution kinetic energy equation to the emitted electrons by ICD decay, with a small variation in the wave packet form of the transition for the final states, with non-Hermitian time-dependent theory.
\end{abstract}

Keywords: decay, ICD, transition

\section{THE PROCESS DESCRIPTION}

The ICD process was predicted by Cederbaum and coworkers [1] and experimental evidence was observed in the recent work of Jahnke et al. [2]. This new type of transition, not accompanied by emission of radiation, starts from the ionization of a monomer in the neon dimer, for the inner valence (i.v.) at $2 s$ orbital. Initially the system is in the state $\varphi^{0}=\varphi_{n} \varphi_{i}$, which is a nuclear vibrational and an electronic state, within the Born-Oppenheimer approximation. Following, one $2 p$ electron of the outer valence (o.v.) shells of the same monomer, occupies the hole in the $2 s$ orbital releasing a certain amount of energy that is not sufficient to ionize the same monomer. However, this energy released is sufficient to cause the emission of another $2 p$ electron from the closest neighboring monomer, which the dimer is composed of, producing two $N e^{+}\left(2 p^{-1}\right)$ ions, which repel each other in a so-called Coulombic explosion. A discussion of the energies involved in process can be seen in Dias [3]. The energy transfer between monomers is strongly connected with the nuclear separation [4]. The process can be described by the sets:

$$
\begin{aligned}
& \hbar w+N e_{2} \Rightarrow\left(N e_{2}^{+(i . v .)}\right)^{*}+e^{-} \Rightarrow\left(N e_{2}^{++(o . v .)}\right) \\
& +e_{I C D}^{-}+e^{-} \Rightarrow 2\left(N e^{+(o . v .)}\right)+e_{I C D}^{-}+e^{-}
\end{aligned}
$$

\section{KINETIC ENERGY DISTRIBUTION EQUATION}

Consider the ICD process [5] as a transition in two times: $i \rightarrow k, k \rightarrow f$. Here, the $(k)$ state is a intermediate decay state; $(i)$ is the vibrational initial state and $(f)$ is the vibrational final state. The initial state $(i)$, it is governed by the wave packet $\varphi^{0}$, eigenstate of the Hermitian operator $\hat{H}^{0}$.

The system is ionized by the inner valence (i.v.) of one of his monomers, leading to a electronic decay state $(k)$, with a wave function $\varphi_{n}^{k}(t)=e^{-i E^{k}} \hat{D} \varphi^{0}$, eigenstate of the nonHermitian operator $\hat{H}^{k}$, governed by a complex potential according to Moiseyev [6]. The operator $\hat{D}(R)$ is the ionization

*Electronic address: alexandre.dias@unifenas.br or dipole operator. Gradually, this wave packet decays to the final state. To each time interval $d t$, part of the packet decays to the final state following the emission of an electron with the $E_{\text {Kin }}$ kinetic energy. Fig. 1 illustrates the decay process described.

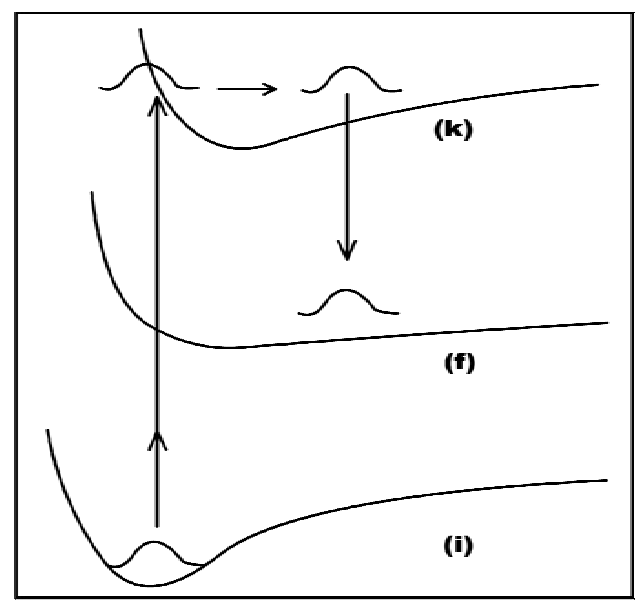

FIG. 1: The ICD transition process

Consider that the decaying wave packet to the final $(f)$ state, has the following form, that differs by only a phase factor of the Cederbaum proposal [5]:

$$
-i \hat{W} \varphi_{n}^{k}(t) \partial t
$$

Here, the operator $\hat{W}(R)$ is the transition operator. With this, it is possible to build a differential general equation for the wave packet in the final $(f)$ state. Consider that this wave packet, at time $t$, have the form $\varphi^{f}(t)=e^{-i\left(H^{f}+E_{K i n}\right) t} \varphi^{f}$, with the time-dependent phase factor $e^{-i\left(H^{f}+E_{K i n}\right) t}$. After $d t$ time interval, this wave packet will be

$$
\begin{aligned}
\partial\left(e^{-i\left(H^{f}+E_{K i n}\right) t} \varphi^{f}\right) & =-i\left(H^{f}+E_{K i n}\right) e^{-i\left(H^{f}+E_{K i n}\right) t} \varphi^{f} \partial t \\
& =-i\left(H^{f}+E_{K i n}\right) \varphi^{f}(t) \partial t
\end{aligned}
$$

Meantime, in the same $d t$ time interval, part of wave packet that decays from intermediate $(k)$ state, assumed in 
equation (1), appears in the surface potential of the final state. So, we have to add this contribution to obtain the total changes in the final wave packet $\varphi^{f}(t)$, as

$$
\partial \varphi^{f}(t)=-i\left(H^{f}+E_{K i n}\right) \varphi^{f}(t) \partial t-i \hat{W} \varphi_{n}^{k}(t) \partial t
$$

This is the general differential equation of the process [7], presented first in the Cederbaum et al. [5] work about the nuclear dynamics of decaying states, which is read as,

$$
i \frac{\partial \varphi^{f}(t)}{\partial t}=\hat{W} \varphi_{n}^{k}(t)+\left(\hat{H}^{f}+E_{K i n}\right) \varphi^{f}(t)
$$

Now, by the same way used in Moiseyev [7] work, the derivation of the distribution energy equation for the emitted electrons in the process will be shown, from this general equation.

Consider the following expansion of the wave final packet $\varphi^{f}(t)$ in the basis set of the eigenfunctions of Hermitian $\hat{H}^{f}$, with the phase factor time-dependent $e^{-i\left(E_{m}^{f}+E_{K i n}\right) t}$, as

$$
\varphi^{f}(t)=\sum_{m} c_{m}(t) e^{-i\left(E_{m}^{f}+E_{K i n}\right) t} \varphi_{m}^{f}
$$

Multiplying from the left by $\varphi^{f *}(t)$, using the relation $<$ $\varphi_{m}^{f} \mid \varphi_{j}^{f}>=\delta_{m, j}$, integrating and taking the limit $t \rightarrow \infty$, we have that

$$
\lim _{t \rightarrow \infty}<\left.\varphi^{f}\left|\varphi^{f}>=\sum_{j}\right| c_{j}(\infty)\right|^{2}
$$

Now, using the expression of the wave function to the intermediate $(k)$ state, $\varphi_{n}^{k}(t)=e^{-i E^{k} t} \hat{D} \varphi^{0}$, the expansion of the final state wave function from equation (5) and substituting into equation (4), it gives

$$
\begin{aligned}
& i \frac{\partial}{\partial t}\left(\sum_{m} c_{m}(t) e^{-i\left(E_{m}^{f}+E_{K i n}\right) t} \varphi_{m}^{f}\right)=\hat{W} e^{-i E^{k} t} \hat{D} \varphi^{0} \\
& +\left(\hat{H}^{f}+E_{K i n}\right) \sum_{m} c_{m}(t) e^{-i\left(E_{m}^{f}+E_{K i n}\right) t} \varphi_{m}^{f}
\end{aligned}
$$

Solving the left derivative, the equation take the form

$$
i \sum_{m}\left(\frac{\partial}{\partial t} c_{m}(t)\right) e^{-i\left(E_{m}^{f}+E_{K i n}\right) t} \varphi_{m}^{f}=\hat{W} e^{-i E^{k} t} \hat{D} \varphi^{0}
$$

Now, multiplying from the left by $e^{i\left(E_{m}^{f}+E_{K i n}\right) t}$, we have that

$$
i \sum_{m}\left(\frac{\partial}{\partial t} c_{m}(t)\right) \varphi_{m}^{f}=\hat{W} e^{i\left(E_{m}^{f}+E_{K i n}-E^{k}\right) t} \hat{D} \varphi^{0}
$$

Following, multiplying from the left by $\varphi_{j}^{f *}$ and integrating for all space, we have

$$
\frac{\partial c_{j}(t)}{\partial t}=i<\varphi_{j}^{f}|\hat{W}| e^{i\left(E_{j}^{f}+E_{K i n}-E^{k}\right) t} \hat{D} \varphi^{0}>
$$

The operator $H^{k}$ is non-Hermitian. So, from this point on, it is important to use the inner product-c, (.|.) as defined by Moiseyev [6]. Consider that, for eigenfunctions of the nonHermitian operator $H k$ we have identities

$$
\left.\mid \psi)=\sum_{n} \mid \varphi_{n}^{k}\right)\left(\varphi_{n}^{k} \mid \psi\right) \quad \text { and } \quad\left(\psi \mid=\sum_{n}\left(\psi \mid \varphi_{n}^{k}\right)\left(\varphi_{n}^{k} \mid\right.\right.
$$

So, we can modify the equation (10) and integrating in the time, to obtain

$\int_{0}^{\infty} d t \frac{\partial c_{j}(t)}{\partial t}=i \sum_{n}\left(\varphi_{j}^{f}|\hat{W}| \varphi_{n}^{k}\right)\left(\varphi_{n}^{k}|\hat{D}| \varphi^{0}\right) \int_{0}^{\infty} d t e^{i\left(E_{j}^{f}+E_{K i n}-E^{k}\right) t}$

and, finally,

$$
c_{j}(\infty)-c_{j}(0)=\sum_{n} \frac{\left(\varphi_{j}^{f}|\hat{W}| \varphi_{n}^{k}\right)\left(\varphi_{n}^{k}|\hat{D}| \varphi^{0}\right)}{\left(E_{j}^{f}-E_{\text {Kin }}\right)-E^{k}}
$$

Taking $c_{j}(0)=0$, because the initial state $(t=0)$ is a stationary state and substituting into equation (6), we have

$$
\lim _{t \rightarrow \infty}<\left.\varphi_{j}^{f}\left|\varphi_{j}^{f}>=\sum_{j}\right| \sum_{n} \frac{\left(\varphi_{j}^{f}|\hat{W}| \varphi_{n}^{k}\right)\left(\varphi_{n}^{k}|\hat{D}| \varphi^{0}\right)}{\left(E_{j}^{f}-E_{K i n}\right)-E^{k}}\right|^{2}
$$

which is the same equation for the energy distribution $\sigma\left(E_{K i n}\right)$ also quoted in Moiseyev et al.[7], obtained by timeindependent scattering theory.

Here, the inner product-c or simply product-c, $(f \mid g)=$ $\int f g d \vec{r}$, defined by Moiseyev [6], substitutes the scalar product, $\langle f \mid g\rangle=\int f^{*} g d \vec{r}$, of quantum mechanics, because the Hamiltonian non-Hermitian operator of the intermediate electronic decay state is governed by complex potential. The function $\mid$.$) is the right eigenvalues, and (.|function, the left$ eigenvalues. As the Hamiltonian $\hat{H}^{f}$ is Hermitian, $\left(\varphi_{j}^{f} \mid=<\right.$ $\varphi_{j}^{f} \mid$, but to eigenfunctions of the non-Hermitian operator of intermediate state, $\hat{H}^{k},\left(\varphi_{n}^{k}\left|\neq<\varphi_{n}^{k}\right|\right.$ or yet $\left(\varphi_{n}^{k}\left|=<\varphi_{n}^{k *}\right|\right.$.

\section{FINAL REMARKS}

We notice that, from equation (10) to equation (12), we used the equivalences $\left(\varphi_{j}^{f}\left|=<\varphi_{j}^{f}\right|\right.$ and $\left.\mid \varphi_{n}^{k}\right)=\mid \varphi_{n}^{k}>$, whereas $\left(\varphi_{n}^{k}\left|=<\varphi_{n}^{k *}\right|\right.$. These substitutions are important for the validity of the demonstration. The equation (12) obtained has the same aspect as the Kramers-Heinsenberg formula, also quoted in Moiseyev [7], that describes the resonant xrays emission, where the intermediate states has eigenvectors and eigenvalues of the Hamiltonian Hermitian.

The Kramers-Heisenberg formula was obtained from second order perturbation theory of the Hermitian quantum mechanics. Finally, we notice that the small modification in the wave function form described in equation (1) leads to the general equation (4) in a direct way. 
[3] Dias, A.M., Physica B: Condensed Matter, 403 (2008), 34903494. doi:10.1016/j.physb.2008.05.014

[4] Scheit, S; Meyer, H-D; Cederbaum, L. S., J. of Physics-Conf. Series 4 (2005), 277-281.

[5] Cederbaum, L. S.; Tarantelli, F. J. Chem. Phys., 98 (1993), 9691.
[6] Moiseyev, N., Quantum Theory of Resonances: Calculating Energies, Widths and Cross-sections by Complex Scaling, Oxford, Elsevier, 1998

[7] Moiseyev, N. et al., J. Chem. Phys., 121 (2004), 722. 